\title{
Effects of age on the association between pulmonary tuberculosis and lung cancer in a South Korean cohort
}

\author{
Soo Jeong $\mathrm{An}^{1}$, Young-Ju Kim², Seon-Sook Han ${ }^{3,4}$, Jeongwon Heo ${ }^{3,4}$ \\ ${ }^{1}$ Department of Benefits Strategy, National Health Insurance Service, Wonju, Republic of Korea; ${ }^{2}$ Department of Statistics, Kangwon National \\ University, Chuncheon, Republic of Korea; ${ }^{3}$ Department of Internal Medicine, School of Medicine, Kangwon National University, Chuncheon, \\ Republic of Korea; ${ }^{4}$ Department of Internal Medicine, Kangwon National University Hospital, Chuncheon, Republic of Korea \\ Contributions: (I) Conception and design: All authors; (II) Administrative support: YJ Kim, SS Han, J Heo; (III) Provision of study materials or \\ patients: SS Han, J Heo; (IV) Collection and assembly of data: SJ An, YJ Kim; (V) Data analysis and interpretation: All authors; (VI) Manuscript \\ writing: All authors; (VII) Final approval of manuscript: All authors. \\ Correspondence to: Jeongwon Heo, MD. Department of Internal Medicine, Kangwon National University Hospital, Baekryeong-ro, Chuncheon, \\ Kangwon-do, 24341, Republic of Korea. Email: doctorhjw@naver.com.
}

Background: Various studies have investigated the association between pulmonary tuberculosis (TB) and lung cancer However, how the relationship between TB and lung cancer may differ by age is not yet clear. This study investigated how risk for lung cancer after pulmonary TB may differ by age.

Methods: This study used the National Health Insurance Service-National Sample Cohort in South Korea. We compared 3,776 pulmonary TB patients with 18,880 controls matched for sex and age during the period from 2003 to 2013. We analyzed the incidence of lung cancer after diagnosis of active pulmonary TB. A multivariate Cox proportional hazard model was used to calculate the adjusted hazard ratio (HR) of lung cancer after adjusting for sex, age, house income, and smoking status.

Results: Among 3,776 pulmonary TB patients, 86 had lung cancer diagnoses, whereas there were 108 lung cancer patients among 18,880 controls. The incidence rate ratio in the pulmonary TB group was 12.26 within 1 year and 3.33 at $1-3.9$ years after TB infection, compared to the control group. There was increased risk for lung cancer in pulmonary TB patients compared to controls (HR, 4.18; 95\% CI, 3.15-5.56). Compared to patients $<50$ years of age, the risks for lung cancer were HR 9.85, 7.1, 3.32, and 2.57 in patients aged 50-59, 60-69, and $\geq 70$ years, respectively.

Conclusions: Pulmonary TB is a risk factor for lung cancer. Patients with pulmonary TB should be monitored for subsequent development of lung cancer, particularly in younger patients.

Keywords: Tuberculosis; lung cancer; cohort study

Submitted Jul 19, 2019. Accepted for publication Jan 08, 2020.

doi: $10.21037 /$ jtd.2020.01.38

View this article at: http://dx.doi.org/10.21037/jtd.2020.01.38

\section{Introduction}

Lung cancer exhibits a high incidence and causes considerable mortality worldwide (1). Epidemiological studies regarding the incidence of lung cancer have supported a possible association between other lung diseases and lung cancer (2-9). Existing lung diseases such as asthma, pneumonia, chronic obstructive pulmonary disease, and pulmonary tuberculosis (TB) may increase the risk for lung cancer, and many studies have sought to clarify these relationships (10-13).

Pulmonary TB is an infectious disease with a high global disease burden. TB incidence in South Korea remains high relative to that of other high-income countries (14). Pulmonary TB is a chronic inflammatory reaction of pulmonary parenchyma that causes irreversible fibrosis and scarring, accompanied by an impaired immune response. It has been hypothesized that the pathological mechanism of 
pulmonary TB increases the risk for lung cancer $(3,5,8,9)$. Several epidemiologic studies have supported a causal link between pulmonary TB and the incidence of lung cancer, but none have investigated whether pulmonary TB increases the incidence of lung cancer in an age-related manner (7-9).

Studies on the effects of pulmonary TB are important in that they can provide epidemiological evidence for the prevention of disease occurrence and support national efforts to manage pulmonary TB. In this study, we investigated how risk for lung cancer after pulmonary TB may change according to age using Korean cohort data.

\section{Methods}

We used data from the National Health Insurance ServiceNational Sample Cohort (NHIS-NCS), which is a population-based cohort. A representative sample cohort was randomly selected of approximately 1 million people, comprising $2.2 \%$ of the entire South Korean population in 2002. The sampling method was systematic stratified random sampling with proportional allocation within each stratum. The cohort was followed for 11 years until 2013. The cohort data included demographic information, as well as data on medical treatments, prescriptions, and general health examinations (15).

The TB group included all patients aged $\geq 20$ years in 2003 who had a new diagnosis of pulmonary TB [International Classification of Diseases code (ICD)- 10 A15, A16, A19] during the period from 2003 to 2013. And it was further confirmed whether two or more TB treatments were prescribed for $>28$ days within 90 days after the first diagnosis of tuberculosis. We excluded subjects who had a diagnosis of TB but did not meet the medication criterion. The latent tuberculosis (LTBI) was defined with the ICD10 R76. To ensure included patients were only those with new-onset $\mathrm{TB}$, we excluded subjects who had a diagnosis of TB in 2002 and those who had a past history of TB. Similarly, we excluded subjects who had a diagnosis of lung cancer (ICD-10 C33, C34) in 2002. Finally, we excluded subjects who had lung cancer before they were diagnosed with TB to focus on the temporal relationship between $\mathrm{TB}$ and development of lung cancer. The index date of TB patients was defined as the earliest recorded TB diagnosis between 2003 and 2013. The control group consisted of five control subjects for each case of TB, matched for sex and age; these control subjects had no records of TB during the period from 2002 to 2013. The same index date was assigned to each of the matched controls. All subjects were followed up from the index date until the development of lung cancer, death, or the end of the year 2013 (Figure 1).

The sex, age, household income, and smoking status of each subject were acquired from the NHIS-NCS database for analyses of factors potentially associated with an increased risk for lung cancer. Age was used to stratify the subjects into four groups at the time of enrollment in the study: $<50,50-59,60-69$, and $\geq 70$ years. Household income levels were used to stratify the subjects into three groups at the time of enrollment in the study: lower $(30 \%)$, middle (40\%), higher (30\%). Smoking statuses (current, former, or never) were obtained from the most recent health examinations at the time of enrollment.

To compare the risk for lung cancer according to the interval since TB diagnosis, follow-up periods were defined as follows: lung cancer development within 1 year after TB diagnosis, 1-3.9 years after TB diagnosis, 4-6.9 years after TB diagnosis, and $\geq 7$ years after TB diagnosis. To compare the risk for lung cancer within $1-3.9$ years after TB diagnosis between subjects in the TB and control groups, we excluded subjects who developed lung cancer or died, as well as those who were diagnosed with TB within 1 year before the end of the study.

We used Pearson's chi-square test to examine differences in characteristics between TB and control groups. The Cox proportional hazards model was used to calculate the crude and adjusted hazard ratios (HRs) of lung cancer; adjustments included sex, age, household income, and smoking status. Survival time was defined as time from index date until lung cancer development, death, or the end of the year 2013. The incidence rate ratios (IRRs) and adjusted HRs were compared according to the interval since TB development. Age-adjusted HRs for lung cancer after TB diagnosis were obtained by stratification based on the age at enrollment in the study. SAS software (version 9.4; SAS Institute Inc., Cary, NC, USA) was used for statistical analyses. The study was reviewed by the Institutional Review Board of Kangwon National University Hospital (IRB No. KNUH-2016-05005).

\section{Results}

A total of 3,776 patients were diagnosed with pulmonary $\mathrm{TB}$ and received at least two anti-TB medications during the study period. Table 1 shows the basic characteristics of the matched subjects. The TB group had low household income $(\mathrm{P}<0.001)$ and a higher prevalence of smoking $(\mathrm{P}=0.024)$. During the study period, 86 of the $3,776 \mathrm{~TB}$ 


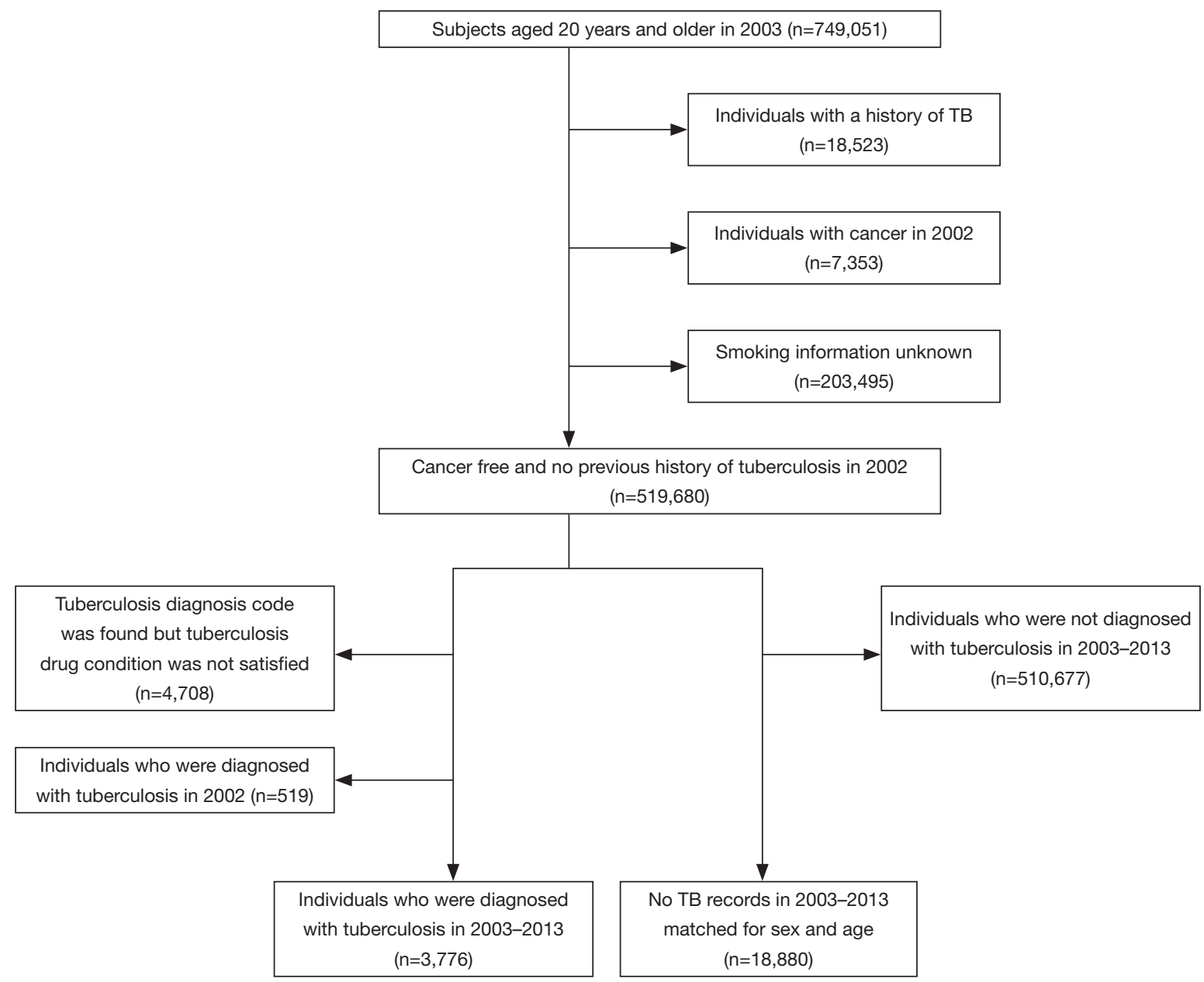

Figure 1 Flow chart of the study selection process.

patients were diagnosed with lung cancer, whereas 108 of the 18,880 controls were diagnosed with it (Tables 2,3). The IRR in pulmonary TB patients was 12.26 within 1 year and 3.33 at $1-3.9$ years after TB diagnosis, compared to the control group. However, there were no statistically significant differences between groups at 4-6.9 years and $\geq 7$ years after TB diagnosis.

There was an increased risk for lung cancer in pulmonary TB patients compared to controls (adjusted HR, 4.18; 95\% CI, 3.15-5.56). Sex, age, and smoking status were all significant risk factors for lung cancer, but there were no significant differences in household income after adjusting for sex, age, smoking status, and TB diagnosis (Table 4). The risk for lung cancer was higher in men than in women (adjusted HR, 2.35; 95\% CI, 1.60-3.46) and in current smokers than in never smokers (HR, 2.00; $95 \%$ CI, 1.43-2.78), and significantly increased with age. Relative to patients $<50$ years of age, the risks were HR 5.67 (95\% CI, 3.46-9.29), 12.76 (95\% CI, 8.31-19.59), and 15.87 (95\% CI, 9.89-25.47) in patients aged 50-59, 60-69, and $\geq 70$ years, respectively (Table 4). However, the HRs after pulmonary TB were 9.85 (95\% CI, 4.57-21.23), 7.14 (95\% CI, 3.63-14.05), 3.32 (95\% CI, 2.12-5.19), 2.57 (95\% CI, 1.40-4.72), respectively. As age increased, the adjusted HR of lung cancer gradually decreased after diagnosis of pulmonary TB (Table 5).

\section{Discussion}

This was a large prospective study of an NHIS-NCS cohort. We compared 3,776 pulmonary TB patients with 18,880 control subjects matched by age and sex and prospectively analyzed the incidence of lung cancer after diagnosis of active pulmonary TB over an 11-year period. 
Table 1 Basic characteristics between cohorts with and without tuberculosis

\begin{tabular}{|c|c|c|c|}
\hline \multirow{2}{*}{ Variables } & \multicolumn{2}{|c|}{ Tuberculosis, n (\%) } & \multirow{2}{*}{$\begin{array}{c}P \\
\text { value }\end{array}$} \\
\hline & Yes & No & \\
\hline Sex & & & 0.999 \\
\hline Female & $1,554(41.15)$ & $7,770(41.15)$ & \\
\hline Male & 2,222 (58.85) & $11,110(58.85)$ & \\
\hline Age group (years) & & & 0.554 \\
\hline$<50$ & 2,178 (57.68) & $10,902(57.74)$ & \\
\hline $50-59$ & $529(14.01)$ & 2,771 (14.68) & \\
\hline $60-69$ & 649 (17.19) & 3,222 (17.07) & \\
\hline$\geq 70$ & $420(11.12)$ & $1,985(10.51)$ & \\
\hline Household income & & & $<0.001$ \\
\hline Lower 30\% & 995 (26.35) & $4,231(22.41)$ & \\
\hline Middle $40 \%$ & $1,513(40.07)$ & $7,186(38.06)$ & \\
\hline Upper 30\% & 1,268 (33.58) & 7,463 (39.53) & \\
\hline Smoking status & & & 0.024 \\
\hline Never smoker & 2,279 (60.35) & $11,837(62.70)$ & \\
\hline Ex-smoker & $321(8.50)$ & $1,542(8.17)$ & \\
\hline Current smoker & $1,176(31.14)$ & $5,501(29.14)$ & \\
\hline Total & 3,776 (16.67) & $18,880(83.33)$ & \\
\hline
\end{tabular}

$P$ value from Pearson's $\chi^{2}$ test.

The HR of lung cancer was 4.18 in pulmonary TB patients compared to controls. Male sex, older age, and current smoking were independent risk factors for the diagnosis of lung cancer. Socioeconomic status was a risk factor in univariate analyses, but this association was lost after adjusting for age, sex, and smoking status. More elderly patients had a diagnosis of lung cancer after TB infection, but younger patients had a higher risk for lung cancer.

Temporal analyses of lung cancer incidence showed that the risk for lung cancer was greatest within 1 year after pulmonary TB diagnosis and was significantly higher within 4 years after diagnosis; however, the risk was similar to that of controls at $>4$ years after diagnosis. This is consistent with $\mathrm{Wu}$ et al., who showed that pulmonary TB was associated with an increased risk for lung cancer in a Taiwanese cohort $(8,9)$. By contrast, Hong et al. analyzed the risk for lung cancer in Korean patients with pulmonary $\mathrm{TB}$, with and without a history of smoking, and reported that risk was independent of TB (16). Unlike the previous studies, we performed analyses on an annual basis, and found that the HR of lung cancer decreased each year after pulmonary TB diagnosis. At $>4$ years after pulmonary TB diagnosis, the risk was not statistically significant, but this might have been affected by the low number of patients. If there was a sufficient number of patients, we believe that higher risk of developing lung cancer in a pulmonary $\mathrm{TB}$ patient would have been significant after 4 years (8).

Pulmonary TB was an independent risk factor for the diagnosis of lung cancer. Diagnostic codes and use of antiTB drugs were used to reduce errors in the identification of pulmonary TB patients. We also included smoking history, which was not included in some cohort studies, and measured the independent risks of lung cancer due to smoking and pulmonary TB, separately.

The association between TB and cancer has attracted attention for several years and remains controversial. Simultaneous occurrence of both TB and cancer in the same organ causes a diagnostic dilemma, as it is difficult to determine whether TB is the cause of lung cancer or whether lung cancer is the cause of TB. The association between TB and cancer can occur in several ways (17). Chronic TB infections cause the accumulation of genomic changes, and exposure to growth factors can cause multistep cellular transformation, leading to dysplasia and malignant squamous cell carcinoma (18). Human susceptibility to TB is also associated with polymorphisms of several genes related to innate immunity and the inflammatory response, such as toll-like receptors, tumor necrosis factors, and inducible nitric oxide; notably, these genes are associated with cancer susceptibility (19-21). We tested whether TB may be the cause of lung cancer, and concluded that a diagnosis of pulmonary TB was a significant risk factor for lung cancer for several years, and that the risk gradually decreased after treatment.

However, pulmonary TB could be a complication of lung cancer, and pulmonary TB and lung cancer may coexist. TB is a known complication in cancer patients. The diagnosis of lung cancer may be delayed when TB and cancer coexist. The risk for lung cancer was greatest within 1 year after diagnosis of pulmonary TB.

We also found that risk for lung cancer was higher in younger patients with pulmonary TB than in elderly patients with pulmonary TB. Both lung cancer and pulmonary TB exhibit age-dependent characteristics and risk factors $(22,23)$. Younger patients with pulmonary TB have common risk factors for both diseases and may develop coexistent lung cancer (17). Wu et al. mentioned that TB 
Table 2 Temporal association of lung cancer incidence between tuberculosis and control patients

\begin{tabular}{|c|c|c|c|c|c|c|c|c|}
\hline Variables & \multicolumn{3}{|c|}{ Tuberculosis $(n=86)$} & \multicolumn{5}{|c|}{ No tuberculosis $(n=108)$} \\
\hline$<1$ year & 50 & 3,711 & $1,347.35$ & 20 & 18,192 & 109.94 & 12.26 & 12.68 (7.54-21.33) \\
\hline $1-3.9$ years & 27 & 8,536 & 316.31 & 42 & 44,168 & 95.09 & 3.33 & $3.42(2.11-5.55)$ \\
\hline $4-6.9$ years & 4 & 5,478 & 73.02 & 30 & 28,581 & 104.96 & 0.70 & $0.73(0.25-2.05)$ \\
\hline
\end{tabular}

Adjusted for sex, age, household income, smoking status. IR, incidence rate; IRR, incidence rate ratio; HR, hazard ratio; CI, confidence interval.

Table 3 Temporal association of lung cancer incidence between tuberculosis and control patients

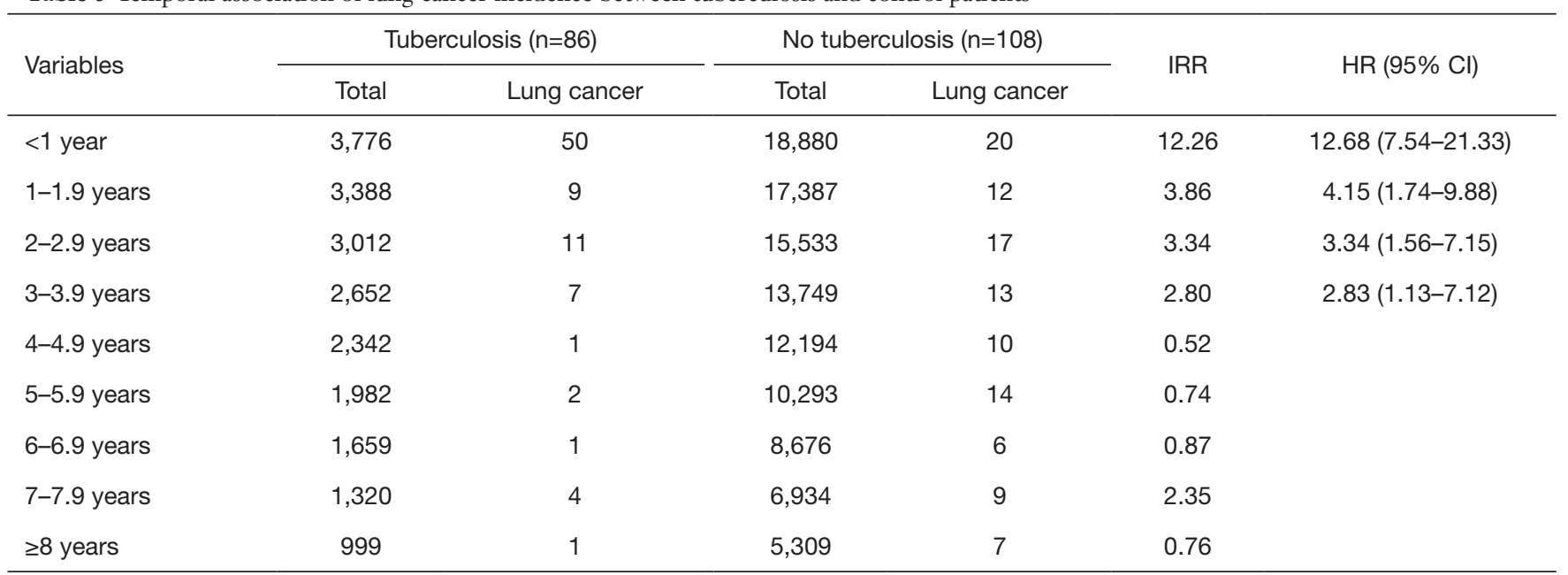

Adjusted for sex, age, household income, smoking status. IR, incidence rate; IRR, incidence rate ratio; $\mathrm{HR}$, hazard ratio; Cl, confidence interval.

plays an important role in the formation of subsequent lung cancer in all age groups, especially in the younger patients. That study reported IRR 3.47 under 60 years old and IRR of 1.55 over 60 years old. But there was no explanation for why (8). We did not find that reason either. Further studies with additional variables, histologic examination, and genetic testing are necessary to fully analyze the risk for lung cancer in young patients with TB.

There were potential limitations to our study. First, we did not consider acid-fast bacilli smear, culture, or imaging information at the time of diagnosis of pulmonary TB (24). In the definition of LTBI for cohort studies, we defined only with the diagnostic code without laboratory screenings, radiological and clinical findings of the patient. However, ICD-10 A16 may include the broad definition of LTBI. Therefore, patients with LTBI who have been treated with two or more TB drugs may have been included as active
TB patients in our study cohort. Second, we did not include information on the histologic results of lung cancer, which could have strengthened the analyses (10). Third, there were no significant results in patients who were followed for more than 4 years because of the lower number of cancer patients (Table 3).

Despite these limitations, our study confirms an association between the diagnosis of lung cancer and diagnosis of pulmonary TB in a large cohort. Stratification by age showed that the risk for lung cancer was greater after diagnosis of pulmonary TB at a younger age. When younger patients are diagnosed with pulmonary TB, they should be more closely monitored for subsequent development of lung cancer than the normal population. Delayed diagnosis of lung cancer due to diagnosis of TB may hinder proper treatment and result in poor prognosis. 
Table 4 Hazard ratio of lung cancer

\begin{tabular}{|c|c|c|c|}
\hline Variables & Univariate, HR (95\% Cl) & Multivariate model 1, HR (95\% Cl) & Multivariate model 2, $\mathrm{HR}(95 \% \mathrm{Cl})$ \\
\hline \multicolumn{4}{|l|}{ Sex } \\
\hline Female & 1.00 & 1.00 & 1.00 \\
\hline Male & $2.45(1.75-3.44)$ & $2.28(1.55-3.34)$ & $2.35(1.60-3.46)$ \\
\hline \multicolumn{4}{|l|}{ Age group (years) } \\
\hline $50-59$ & $5.46(3.34-8.94)$ & $5.62(3.43-9.21)$ & $5.67(3.46-9.29)$ \\
\hline $60-69$ & $10.90(7.13-16.67)$ & $12.65(8.24-19.43)$ & $12.76(8.31-19.59)$ \\
\hline$\geq 70$ & $11.05(6.98-17.51)$ & $15.46(9.64-24.78)$ & $15.87(9.89-25.47)$ \\
\hline \multicolumn{4}{|l|}{ Household income } \\
\hline Upper & $0.71(0.50-0.99)$ & $0.73(0.52-1.03)$ & $0.78(0.56-1.11)$ \\
\hline \multicolumn{4}{|l|}{ Smoking status } \\
\hline Never smoker & 1.00 & 1.00 & 1.00 \\
\hline Ex-smoker & $1.01(0.57-1.81)$ & $0.85(0.46-1.54)$ & $0.80(0.44-1.45)$ \\
\hline Current smoker & $1.89(1.41-2.52)$ & $2.06(1.48-2.87)$ & $2.00(1.43-2.78)$ \\
\hline \multicolumn{4}{|l|}{ Tuberculosis } \\
\hline No & 1.00 & & 1.00 \\
\hline Yes & $4.10(3.09-5.45)$ & & $4.18(3.15-5.56)$ \\
\hline
\end{tabular}

Model 1: adjusted for sex, age, household income, smoking status; Model 2: adjusted for sex, age, household income, smoking status, tuberculosis. HR, hazard ratio; $\mathrm{Cl}$, confidence interval.

Table 5 Hazard ratio of lung cancer related to tuberculosis by age

\begin{tabular}{|c|c|c|c|c|c|}
\hline Age group (years) & Tuberculosis & \multicolumn{2}{|c|}{ Lung cancer, n (\%) } & Univariate, HR (95\% Cl) & Multivariate model $1, \mathrm{HR}(95 \% \mathrm{Cl}$ \\
\hline \multirow[t]{2}{*}{$<50$} & No & $10,892(99.91)$ & $10(0.09)$ & 1.00 & 1.00 \\
\hline & Yes & 2,159 (99.13) & $19(0.87)$ & $9.60(4.46-20.64)$ & $9.85(4.57-21.23)$ \\
\hline $50-59$ & No & $2,756(99.46)$ & $15(0.54)$ & 1.00 & 1.00 \\
\hline \multirow[t]{2}{*}{$60-69$} & No & $3,173(98.48)$ & $49(1.52)$ & 1.00 & 1.00 \\
\hline & Yes & $617(95.07)$ & $32(4.93)$ & $3.49(2.24-5.45)$ & $3.32(2.12-5.19)$ \\
\hline \multirow[t]{2}{*}{$\geq 70$} & No & $1,951(98.29)$ & $34(1.71)$ & 1.00 & 1.00 \\
\hline & Yes & $405(96.43)$ & $15(3.57)$ & 2.35 (1.28-4.32) & $2.57(1.40-4.72)$ \\
\hline
\end{tabular}

Model 1: adjusted for sex, household income, smoking status. HR, hazard ratio; Cl, confidence interval. 


\section{Conclusions}

Pulmonary TB is associated with risk for lung cancer, such that there is a greater risk for lung cancer within 4 years after pulmonary TB. Patients with pulmonary TB should be monitored for subsequent development of lung cancer, particularly in younger patients.

\section{Acknowledgments}

Funding: This study used National Health Insurance Service-National Sample Cohort data (NHIS-2017-2-574), made by National Health Insurance Service.

\section{Footnote}

Conflicts of Interest: The authors have no conflicts of interest to declare.

Ethical Statement: The authors are accountable for all aspects of the work in ensuring that questions related to the accuracy or integrity of any part of the work are appropriately investigated and resolved. The study was reviewed by the Institutional Review Board of Kangwon National University Hospital (IRB No. KNUH-201605-005).

Open Access Statement: This is an Open Access article distributed in accordance with the Creative Commons Attribution-NonCommercial-NoDerivs 4.0 International License (CC BY-NC-ND 4.0), which permits the noncommercial replication and distribution of the article with the strict proviso that no changes or edits are made and the original work is properly cited (including links to both the formal publication through the relevant DOI and the license). See: https://creativecommons.org/licenses/by-nc-nd/4.0/.

\section{References}

1. Bray F, Jemal A, Grey N, et al. Global cancer transitions according to the Human Development Index (2008-2030): a population-based study. Lancet Oncol 2012;13:790-801.

2. Brenner DR, Boffetta P, Duell EJ, et al. Previous lung diseases and lung cancer risk: a pooled analysis from the International Lung Cancer Consortium. Am J Epidemiol 2012;176:573-85.

3. Brenner DR, McLaughlin JR, Hung RJ. Previous lung diseases and lung cancer risk: a systematic review and meta-analysis. PLoS One 2011;6:e17479.

4. Denholm R, Schuz J, Straif K, et al. Is previous respiratory disease a risk factor for lung cancer? Am J Respir Crit Care Med 2014;190:549-59.

5. Liang HY, Li XL, Yu XS, et al. Facts and fiction of the relationship between preexisting tuberculosis and lung cancer risk: a systematic review. Int J Cancer 2009;125:2936-44.

6. Littman AJ, Thornquist MD, White E, et al. Prior lung disease and risk of lung cancer in a large prospective study. Cancer Causes Control 2004;15:819-27.

7. Simonsen DF, Farkas DK, Sogaard M, et al. Tuberculosis and risk of cancer: a Danish nationwide cohort study. Int J Tuberc Lung Dis 2014;18:1211-9.

8. Wu CY, Hu HY, Pu CY, et al. Pulmonary tuberculosis increases the risk of lung cancer: a population-based cohort study. Cancer 2011;117:618-24.

9. $\mathrm{Yu} \mathrm{YH}$, Liao CC, Hsu WH, et al. Increased lung cancer risk among patients with pulmonary tuberculosis: a population cohort study. J Thorac Oncol 2011;6:32-7.

10. Huang JY, Jian ZH, Nfor ON, et al. The effects of pulmonary diseases on histologic types of lung cancer in both sexes: a population-based study in Taiwan. BMC Cancer 2015;15:834.

11. Santillan AA, Camargo CA Jr, Colditz GA. A meta-analysis of asthma and risk of lung cancer (United States). Cancer Causes Control 2003;14:327-34.

12. Turner MC, Chen Y, Krewski D, et al. Chronic obstructive pulmonary disease is associated with lung cancer mortality in a prospective study of never smokers. Am J Respir Crit Care Med 2007;176:285-90.

13. Young RP, Hopkins RJ, Christmas T, et al. COPD prevalence is increased in lung cancer, independent of age, sex and smoking history. Eur Respir J 2009;34:380-6.

14. Kim JH, Yim JJ. Achievements in and Challenges of Tuberculosis Control in South Korea. Emerg Infect Dis 2015;21:1913-20.

15. Lee J, Lee JS, Park SH, et al. Cohort Profile: The National Health Insurance Service-National Sample Cohort (NHIS-NSC), South Korea. Int J Epidemiol 2017;46:e15.

16. Hong S, Mok Y, Jeon C, et al. Tuberculosis, smoking and risk for lung cancer incidence and mortality. Int J Cancer 2016;139:2447-55.

17. Harikrishna J, Sukaveni V, Kumar DP, et al. Cancer and tuberculosis. Journal, India Academy of Clinical Medicine 2012;13:142-4.

18. Nalbandian A, Yan BS, Pichugin A, et al. Lung 
carcinogenesis induced by chronic tuberculosis infection: the experimental model and genetic control. Oncogene 2009;28:1928-38.

19. Azad AK, Sadee W, Schlesinger LS. Innate immune gene polymorphisms in tuberculosis. Infect Immun 2012;80:3343-59.

20. Kutikhin AG. Association of polymorphisms in TLR genes and in genes of the Toll-like receptor signaling pathway with cancer risk. Hum Immunol 2011;72:1095-116.

21. Qidwai T, Khan F. Tumour necrosis factor gene polymorphism and disease prevalence. Scand J Immunol

Cite this article as: An SJ, Kim YJ, Han SS, Heo J. Effects of age on the association between pulmonary tuberculosis and lung cancer in a South Korean cohort. J Thorac Dis 2020;12(3):375382. doi: $10.21037 /$ jtd.2020.01.38
2011;74:522-47.

22. Kozielski J, Kaczmarczyk G, Porębska I, et al. Lung cancer in patients under the age of 40 years. Contemp Oncol (Pozn) 2012;16:413-5.

23. Donald PR, Marais BJ, Barry III CE. Age and the epidemiology and pathogenesis of tuberculosis. Lancet 2010;375:1852-4.

24. Leylabadlo HE, Kafil HS, Yousefi M, et al. Pulmonary tuberculosis diagnosis: where we are? Tuberc Respir Dis (Seoul) 2016;79:134-42. 